\title{
ALMA Observation of Mass Loss from Massive Stars
}

\author{
D. Y. A. Setia Gunawan ${ }^{1}$, M. Curé ${ }^{1}$, S. Kanaan ${ }^{1}$, J. Puls ${ }^{2}$, \\ F. Najarro ${ }^{3}$, J. Sundqvist ${ }^{3}$, M. M. Rubio Diez ${ }^{3}$ and N. Whyborn ${ }^{4}$ \\ ${ }^{1}$ IFA, Universidad de Valparaíso, Av. Gran Bretaña 1111, Valparaíso, Chile \\ ${ }^{2}$ LMU Munich, Universitäts-Sternwarte, Scheinerstr. 1, D-81679 Müchen, Germany \\ ${ }^{3}$ Centro de Astrobiología (CSIC/INTA), 28850 Torrejón de Ardoz, Madrid, Spain \\ ${ }^{4}$ Joint ALMA Observatory, Alonso de Cordova 3107, Vitacura, Santiago, Chile
}

\begin{abstract}
We present a pilot study of using the Atacama Large Millimeter/sub-millimeter Array (ALMA) continuum observations to constrain the density structure in the intermediate wind zone of massive stars, in which the wind is extremely sensitive to clumping.
\end{abstract}

Keywords. Stars, stars: mass loss, stars: early-type, radio continuum: stars, submillimeter

\section{Using ALMA to determine mass loss rates from massive stars}

The fast, dense outflows from massive OB type stars are driven by radiative line acceleration. Multiple evidence points to an over-estimation of the currently accepted massloss rates from these stars as shown by inconsistencies in results derived using different diagnostics, attributed to wind clumping. Potential downward revisions of mass-loss rates of massive stars have a profound effect on the stars' evolution, the feedback from them, and in turn affects the evolution of the host galaxy. We urgently need to establish the true mass-loss rates of OB stars, by constraining the clumping structure. Models predict that the clumping is radially stratified. If the radial stratification of clumping is known from consistent analyses of different diagnostic methods, realistic mass-loss rates can be derived. (Sub-)mm observations of stellar winds will provide the critical diagnostics for the clumping structure in the currently unconstrained intermediate wind regions of massive OB stars, in which the wind is extremely sensitive to clumping. Our pilot study showed that ALMA can efficiently provide the necessary (sub-)millimeter data.

(Sub-)millimeter fluxes of selected bright OB type stars with available multi-wavelength observation result, HD HD37043, HD37128, HD37742, HD38771, HD66811, HD149757, HD151804, and HD152236, were obtained by ALMA between April 2013 - April 2014, using continuum observing mode in the ALMA bands B3, B6 and B7 (respectively at $100,230,345 \mathrm{GHz}$ ). The data was reduced and calibrated using the standard ALMA reduction scripts using CASA. Subsequently obtained CLEANed maps have $\mathrm{S} / \mathrm{N}>8$. The (sub-)millimeter SEDs show mostly spectral indices consistent with emission from thermal winds. One target may show a non-thermal emission. Our next step is to combine analysis of all diagnostics from the Far-UV to the radio domain with consistency, to derive the clumping properties throughout the entire wind and help to constrain the physical origin of wind clumping. Expanding the sample and using more ALMA band coverage is necessary to get the complete true mass-loss rate of massive stars. 


\section{Acknowledgement}

This paper makes use of the following ALMA data: ADS/JAO.ALMA\# 2012.1.00941.S (PI Curé) and 2012.1.00955.S (PI Kanaan). ALMA is a partnership of ESO (representing its member states), NSF (USA) and NINS (Japan), together with NRC (Canada), NSC and ASIAA (Taiwan), and KASI (Republic of Korea), in cooperation with the Republic of Chile. The Joint ALMA Observatory is operated by ESO, AUI/NRAO and NAOJ. Este trabajo conta con el apoyo de CONICYT Programa de Astronomia Fondo ALMACONICYT cargo proyectos numeros 31AS002 y 31140024. 